\title{
Energy/bandwidth-Saving Cooperative Spectrum Sensing for Two-hop WRAN
}

\author{
Ming-Tuo Zhou, Chunyi Song, Chin Sean Sum and Hiroshi Harada \\ Smart Wireless Laboratory, NICT \\ Email: mingtuo@nict.com.sg
}

\begin{abstract}
A two-hop wireless regional area network (WRAN) providing monitoring services using Television White Space (TVWS), i.e., IEEE p802.22b, is now under development. A great number of subscriber customer-premises equipments (SCPEs) may be connected to a base station with relaying of a relay CPE (R-CPE) in $\mathbf{p 8 0 2 . 2 2 b}$. Cost-effective and powersaving S-CPEs are needed due to the its large volume and the possibility of absence of main power supply. This paper proposes cooperative spectrum sensing (CSS) employing energy detection to IEEE p802.22b. With the proposal, a number of S-CPEs are instructed to cooperatively sense TV channels and a R-CPE serves as fusion center. We further propose that when an S-CPE without upstream detects the presence of primary users it does not report to the R-CPE if it detects a signal-to-noise ratio (SNR) less than a pre-defined threshold, for purpose of saving energy and bandwidth consumption. Numerical results show that with the proposed energy/bandwidth-saving scheme the fused error probability changes very little while the fused miss-detection probability and false alarm probability are lower than $10 \%$ that is required by 802.22. With $10 \mathrm{~S}$-CPEs participating CSS, it is possible to save more than $40 \%$ of the energy/bandwidth in a Rayleigh fading channel.
\end{abstract}

\section{INTRODUCTION}

With the explosive growth of broadband wireless users and services, the current spectrum for wireless communications becomes more and more congested. Fortunately, to explore Television White Space (TVWS) - the unused TV channels at certain time in certain geographic area - may alleviate the problem. IEEE 802.22 is one of the current efforts to utilize TVWS for services in regional area. The 802.22 working group has developed IEEE Std 802.22-2011 for regional broadband services and now is working on IEEE p802.22b for regional monitoring and metering, etc [1], [2].

Unlike 802.22-2011 that employs cellular network topology, p802.22b incorporates two-hop relays where a great number (e.g., tens to hundreds) of subscriber customer-premises equipments (S-CPEs) may connect to a relay CPE (R-CPE) and then a multi-hop base station (MR-BS). IEEE p802.22b S-CPEs require cost-effective and energy/bandwidth-saving designs due to the big volume and the fact that they may not be main powered.

On the other hand, following regulatory requirement, periodic quiet periods (QPs) are reserved in $\mathrm{p} 802.22 \mathrm{~b}$ frames where an S-CPE can perform spectrum sensing to detect the presence of the primary users (PUs). The current p802.22b employs individual spectrum sensing technologies, some of which require high-performance processors therefore result in high cost at S-CPE [1].
Based on consideration of cost and simplicity design of SCPEs, we propose cooperative spectrum sensing (CSS) for IEEE p802.22b. With CSS, many nodes sense the spectrum at same time and report individual result to a fusion center (FC). The FC compounds the received individual results and makes the final sensing decision. Compared to sensing by a single node, the sensing performance can be improved due to space diversity of the radio signal [7], [8]. It is therefore at each S-CPE, relatively lower sensing performance is required and then lower-cost processor can be used. In the proposed scheme, as shown in Fig. 1, a number of S-CPEs associated with a R-CPE perform CSS and report results to the R-CPE. The R-CPE acts as FC and reports the fused result to the MR-BS it associated with. If a fused result reported by a R$\mathrm{CPE}$ is the presence of the PU, the MR-BS needs to request the R-CPE and the associated S-CPEs to stop transmissions immediately.

Moreover, in order to save energy and bandwidth for a p802.22b system that employs a great number of S-CPEs that may be powered by batteries, we propose only the S-CPEs with detected primary signal-to-noise ratio (SNR) higher than a threshold to report the sensing results to a R-CPE. An SNR threshold is stored at each S-CPEs for for comparison to the detected SNR locally. Numerical results show that with the proposed partial reporting scheme, the energy/bandwidth can be saved while the sensing performance can be maintained. The saved energy/bandwidth increases with increasing the number of the sensing S-CPEs. The principle of the proposal applies to other spectrum sensing technologies although energy detection is considered here for simplicity.

In the literatures [3]-[12], researches of energy/bandwidthsaving for CSS incorporating energy detection focus on two approaches: (1) to reduce energy for individual spectrum sensing; (2) to reduce energy for reporting individual sensing result to an FC. In [9], Chien et al. proposed a partial spectrum sensing technology to save energy for spectrum sensing. In [10], the spectrum sensors are divided into subsets and scheduled to cooperatively sense the spectrum in an optimized sequence so that the overall energy consumption is minimized. [3] and [4] demonstrated that the overall energy for CSS can be saved when only an optimal number of SUs participate CSS and each SU employs optimized sensing time. In [12], Zhang et al. studied the optimal fusion rule and optimized the number of SUs performing CSS. Compared to other proposals, the energy/bandwidth-saving scheme of this study requires no 
global comparison of the primary SNR thus extra energy and spectrum can be saved.

This paper is organized as follows. Following the introduction, we introduce p802.22b in section II. Then we present the proposed cooperative scheme for $\mathrm{p} 802.22 \mathrm{~b}$ in section III. CSS with energy/bandwidth-saving is proposed and analyzed in section IV. Numerical results are presented in section V and the paper is concluded by section VI.

\section{IEEE P802.22B AND SPECTRUM SENSING}

There are various broadband services and monitoring applications in context of wireless regional area networks where 802.22-2011 device may not able to serve. The regional services include real-time and near real-time monitoring, emergency broadband services, remote medical diagnose, etc, where a great number of subscriber terminals with simpler and lower cost are needed but are not supported by 802.222011. For this consideration, IEEE p802.22b is to amend IEEE Std 802.22-2011 by introducing new class CPEs, i.e., R-CPEs and S-CPEs. An S-CPE is of lower capability, for example, lower transmission power, lower antenna height, and lowergain/cost amplifier, etc. Thus the effective communication distance for an S-CPE is not likely to be tens of kilometers, but is of 1 to 2 kilometers. A R-CPE is a 802.22-2011 CPE supporting advanced functions such as relay, multipleinput multiple out (MIMO) and channel bounding, etc. The supported communion distance of a R-CPE is of tens of kilometers. Correspondingly, an MR-BS supports advanced functions like relay, MIMO and channel bounding, etc. As shown in Fig. 1, in p802.22b, the data traffic between the SCPEs and an MR-BS is relayed by the R-CPEs.

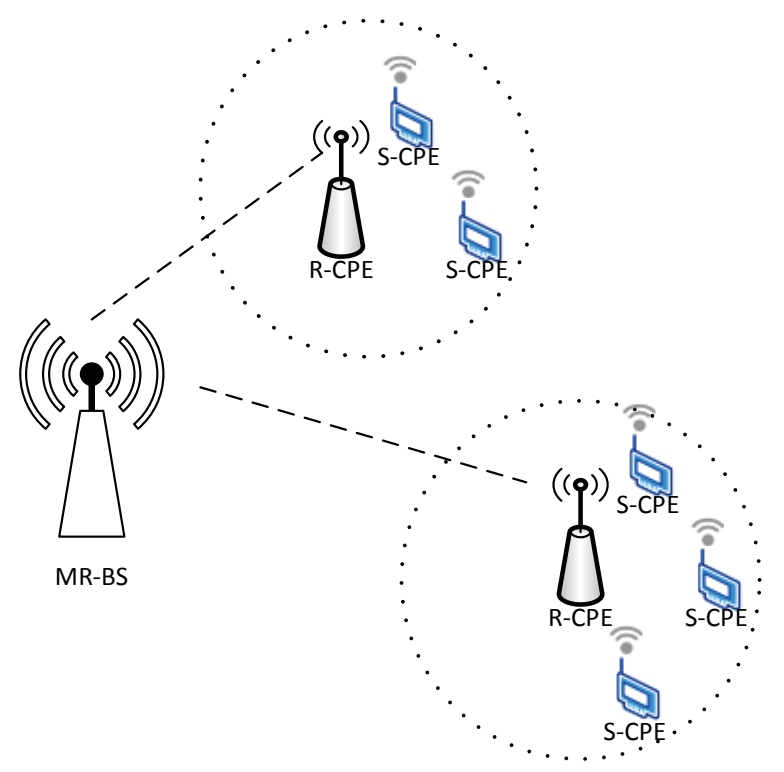

Fig. 1. IEEE p802.22b network structure.

The general frame structure of p802.22b is shown in Fig. 2, where both downstream (DS) subframe and upstream (US) subframe are divided into access zone and relay zone. As shown in the figure, a contention window in both access and relay zone of a US subframe is allocated for ranging, bandwidth request, and urgent coexistence situation (UCS) notification.

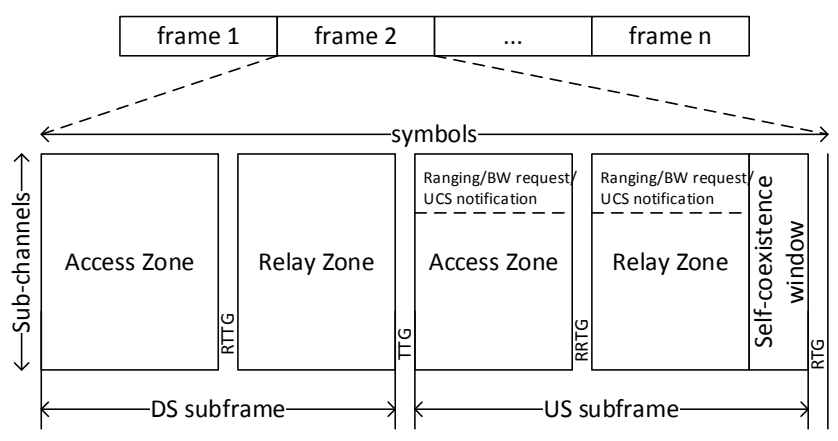

Fig. 2. IEEE p802.22b general frame structure.

Following 802.22-2011, periodic Quiet Periods (QPs) are scheduled along the frames for incumbent detection by spectrum sensing in $\mathrm{p} 802.22 \mathrm{~b}$. It has been designed that across a 802.22 network, QPs at all stations are synchronized. In a 802.22b system, a fraction of the S-CPEs/R-CPEs can be instructed to sense the spectrum and if the presence of PUs are detected, it needs to be reported to a BS as soon as possible.

In current p802.22b, an S-CPE has two possible ways to report the detected results. For an S-CPE with upstream bandwidth allocation, it sets the UCS flag in the generic MAC header for reporting to a BS/MR-BS. For an S-CPE without upstream bandwidth allocation, it needs to report in the UCS window using contention or code-division multiplexing access (CDMA).

\section{COOPERATIVE SPECTRUM SENSING FOR P802.22B}

\section{A. Proposal of CSS}

The big volume of S-CPEs under a R-CPE imposes requirement of simplicity and low-cost design for an S-CPE. To meet this requirement, we propose CSS in p802.22b. It is well known that by CSS, the overall sensing performance can be improved and then the requirement of individual sensing performance can be relaxed. Therefore an S-CPE may employs a simpler and low-cost sensing component thus the total cost can be saved.

With the proposed CSS, under a R-CPE, a number of SCPEs are instructed to sense the spectrum cooperatively. After a QP during which spectrum sensing is performed, a S-CPE reports to a R-CPE if the presence of PU is detected at its location. The R-CPE acts as fusion center and reports to an MR-BS if the presence of PU is detected. OR-fusion rule is used and energy detection sensing is employed since compared to other sensing technology it is relatively simple and low-cost. Of course, CSS proposed in this study is not limited to energy detection sensing. If other high performance individual sensing is applied at all S-CPEs, it is also possible to apply CSS. 


\section{B. system model and assumptions}

Referring to the network structure shown in Fig. 1, it assumes that under a R-CPE, $N$ S-CPEs are instructed to sense spectrum during any QP. Among the $N$ S-CPEs, $g \cdot N$ S-CPEs have been allocated upstream bandwidth, and $(1-g) \cdot N \mathrm{~S}$ CPEs have no upstream bandwidth, where $0 \leq g \leq 1$. If an S-CPE detects the presence of PU during a QP, then it reports to the R-CPE immediately, otherwise it does not report. If a $\mathrm{R}-\mathrm{CPE}$ receives a report from an $\mathrm{S}-\mathrm{CPE}$, then it assumes the $\mathrm{S}-\mathrm{CPE}$ have detected the presence of PU, otherwise it assumes that the S-CPE detects no presence of PU.

For presentation simplicity, the S-CPEs with upstream bandwidth are called Group-1 S-CPEs and the S-CPEs without upstream bandwidth are called Group-2 S-CPEs, since their reporting mechanisms are different. For Group-1 S-CPEs, it assumes all S-CPEs can report successfully as they have upstream bandwidth. For Group-2 S-CPEs, contention/CDMA reporting method is used as of current $802.22 \mathrm{~b}$, in case there are two or more S-CPEs to report, an S-CPE may fail to report. Clearly, when more Group-2 S-CPEs report in an UCS window, the success probability will be lower. It is difficult to precisely describe the relationship between the success reporting probability and the number of the reporting S-CPEs mathematically due to the complexity. In this study, we assume the success reporting probability is substantially modeled by following equation

$$
p_{t}(X)=e^{-X / 10}, \quad X=2,3, \ldots,
$$

where $X$ is the number of Group-2 S-CPEs reporting in same UCS window.

If a Group-2 S-CPE detects the presence of PU but it fails in reporting, then the R-CPE assumes that S-CPE has not detected the presence of PU.

We assume that the detected instantaneous signal-to-noise ratio (SNR) of the primary signal at each S-CPE varies with time due to channel fading, and the averaged SNR $\bar{\gamma}$ at all SCPEs under a R-CPE are equal since the path loss exponent of the $\mathrm{p} 802.22 \mathrm{~b}$ scenarios is general low and the distance between the S-CPEs of a local cell is in range of 1 to 2 kilometers, which is generally much shorter than the distance to a primary transmitter (usually a TV tower).

Let $p_{d}$ and $p_{f}$ stand for the local detection probability and false alarm probability at an S-CPE, respectively. For fading channel, $p_{d}$ and $p_{f}$ are averaged over the primary SNR.

High detection probability and low false alarm probability are required by $\mathrm{p} 802.22 \mathrm{~b}$ for effective protection of PUs and utilization of TVWS, respectively. Without loss of generality, for the proposed CSS scheme to $\mathrm{p} 802.22 \mathrm{~b}$, we propose that at a R-CPE, the fused detection probability $Q_{d}$ should be higher than $90 \%$ and the fused false alarm probability $Q_{f}$ should be lower than $10 \%$. The fused miss-detection probability, i.e., $Q_{m}$ $\left(=1-Q_{d}\right)$ therefore should be less than $10 \%$.

\section{C. fused miss-detection probability}

The fused miss-detection probability at a R-CPE is given by $Q_{m}=Q_{m_{1}} \cdot Q_{m 2}$, where $Q_{m_{1}}$ is fused miss-detection probability of the Group-1 S-CPEs and $Q_{m_{2}}$ is fused missdetection probability of the Group-2 S-CPEs.

Let $H_{1}$ stands for the assumption that PUs are present during sensing, and $H_{0}$ stands for the counterpart, i.e., no PU is present.

With assumption of $H_{1}$, we have

$$
Q_{m_{1}}=\left(1-p_{d}\right)^{g N}
$$

and

$$
\begin{gathered}
Q_{m_{2}}=\left(1-p_{d}\right)^{(1-g) N}+\sum_{K=2}^{(1-g) N}\left\{\left(\begin{array}{c}
(1-g) N \\
K
\end{array}\right) \times,\right. \\
\left.\left\{p_{d}\left[1-p_{t}(K)\right]\right\}^{K}\left(1-p_{d}\right)^{[(1-g) N-K]}\right\}
\end{gathered}
$$

where $K$ is the number of Group-2 S-CPEs detected $H_{1}$. In Eq. 3, the first term corresponds to none of the Group-2 SCPEs have detected the presence of the primary signal, for the second term, there are $K$ Group-2 S-CPEs have detected PU signal, however, none of them succeeded to report to the R-CPE due to conflicts. Note $K$ is in range of [2, $(1-g) N]$. When only one S-CPE detects PU signal, it can report to RCPE successfully since there is no competitors.

\section{D. fused false alarm probability}

The fused false alarm probability at a R-CPE is given by $Q_{f}=1-\left(1-Q_{f_{1}}\right) \cdot\left(1-Q_{f_{2}}\right)$, where $Q_{f_{1}}$ is fused false alarm probability of the Group-1 S-CPEs and $Q_{f_{2}}$ is fused false alarm probability of the Group-2 S-CPEs.

With assumption of $H_{0}$, we have

$$
Q_{f_{1}}=1-\left(1-p_{f}\right)^{g N}
$$

and

$$
\begin{aligned}
Q_{f_{2}} & =1-\left(1-p_{f}\right)^{(1-g) N}-\sum_{L=2}^{(1-g) N}\left\{\left(\begin{array}{c}
(1-g) N \\
L
\end{array}\right) \times,\right. \\
& \left.\left\{p_{f}\left[1-p_{t}(L)\right]\right\}^{L}\left(1-p_{f}\right)^{(1-g) N-L}\right\}
\end{aligned}
$$

where $L$ is the number of Group-2 S-CPEs detected $H_{1}$ (false alarm).

\section{E. fused error probability}

The fused error probability at a R-CPE is given by

$$
Q_{e}=P_{1} Q_{m}+P_{0} Q_{f},
$$

where $P_{1}$ is the probability of $H_{1}$ and $P_{0}$ is probability of $H_{0}$. Clearly, when $Q_{m}\left(=1-Q_{d}\right)$ and $Q_{f}$ must be lower than $10 \%, Q_{e}$ should be less than $10 \%$.

\section{ENERGY/BANDWIDTH-SAVING CSS FOR P802.22B}

The main mechanism of the proposed energy/bandwidthsaving CSS for p802.22b is to exclude the S-CPEs with ignorable contribution to the sensing performance from reporting their sensing results. By this way, power can be saved and conflicts in reporting sensing results to R-CPEs can be reduced. 


\section{A. energy detection}

With spectrum sensing method of energy detection, an S$\mathrm{CPE}$ collects energy during sensing window and the collected energy is compared to a threshold. If the collected energy is bigger, it decides $H_{1}$, otherwise, it decides $H_{0}$. The local false alarm probability and miss-detection probability are given, respectively, by [13]

$$
\begin{aligned}
& p_{f, i}=\operatorname{Pr}\left\{E_{i}>\lambda_{i} \mid H_{0}\right\}=\Gamma\left(u, \lambda_{i} / 2\right) / \Gamma(u), \\
& p_{d, i}=\operatorname{Pr}\left\{E_{i}>\lambda_{i} \mid H_{1}\right\}=Q_{u}\left(\sqrt{2 \gamma_{i}}, \sqrt{\lambda_{i}}\right),
\end{aligned}
$$

where $E_{i}, \lambda_{i}$, and $\gamma_{i}$ are the collected energy, the energy threshold, and the instantaneous detected SNR at the $i$ th SCPE, respectively; $u$ is the sensing time-bandwidth product and is assumed an integer for simplicity. $\Gamma(\cdot)$ and $\Gamma(m, x)$ are the complete and incomplete gamma function, respectively; $Q_{m}(a, x)$ is the generalized Marcum Q-function.

We assume that all S-CPEs have same energy threshold, i.e., $\lambda_{i}=\lambda$, then all S-CPEs have equal false alarm probability, i.e., $p_{f}$. For fading channel, the averaged detection probability, i.e., $p_{d}$ is given by $p_{d}=\int_{0}^{\infty} Q_{u}(\sqrt{2 x}, \sqrt{\lambda}) f_{\gamma}(x) d x$, where $f_{\gamma}(\cdot)$ is probability density function of the primary SNR $(\gamma)$.

\section{B. proposed energy/bandwidth saving CSS}

Since at each round of spectrum sensing, the detection probability $p_{d, i}$ is increasing function of the instantaneous primary SNR, it is possible to exclude S-CPEs that have relatively low instantaneous SNR from reporting its result to a R-CPE, in condition that the deterioration of the fused detection probability still meets the sensing requirement. Estimation of SNR at a receiver has been reported in [15], [14]. By excluding such S-CPEs to report results if they detect $H_{1}$, it also helps to reduce the fused false alarm probability and increase the success reporting probability. By doing so, energy consumption and bandwidth can be saved in a $802.22 \mathrm{~b}$ system.

We propose to set a SNR threshold $\gamma_{t}$, and if a Group-2 SCPEs detected an instantaneous SNR smaller than $\gamma_{t}$, in this round of sensing, it does not report to a R-CPE even it detects $H_{1}$. This is not so meaningful to Group-1 S-CPEs since they have been granted bandwith and will be likely to transmit packages.

In other studies, it has been also found that optimal CSS performance can be achieved if only nodes with the highest SNRs to report for final fusion [4]. However, to select the nodes with 'the highest SNR' global comparison is needed. The scheme proposed in this section requires only local comparison to $\gamma_{t}$ and no global comparison needed thus it may save more energy and bandwidth.

Let $\hat{p}=\int_{0}^{\gamma_{T}} f_{\gamma}(x) d x$ stands for the probability that a detected instantaneous SNR is less than the SNR threshold, then the fused miss-detection probability of Group-2 S-CPEs and fused false alarm probability of Group-2 S-CPEs at a RCPE are given by Eq. 9 and Eq. 10, respectively, where $I$ and $J$ are the number of S-CPEs that have detected $H_{1}$ but the detected SNRs are less than $\gamma_{T}, M$ and $L$ are the number of S-CPEs that have detected $H_{1}$ and the detected SNR are higher than $\gamma_{T}$ while they failed to report the results to the R-CPE.

The fused miss-detection probability and false alarm probability at a R-CPE are given by $Q_{m}^{*}=Q_{m_{1}} \cdot Q_{m_{2}}^{*}$ and $Q_{f}^{*}=1-\left(1-Q_{f_{1}}\right) \cdot\left(1-Q_{f_{2}}^{*}\right)$, respectively. The error probability at a R-CPE becomes $Q_{e}^{*}=P_{1} Q_{m}^{*}+P_{0} Q_{f}^{*}$.

As a higher SNR threshold allows more energy saved, to find the maximum allowed SNR threshold $\gamma_{T}^{*}$ needs to maximize $Q_{m}^{*}$ and $Q_{f}^{*}$ within their upper bounds. Following the requirement of 802.22 system, $Q_{m}^{*}$ and $Q_{f}^{*}$ should be less than $10 \%$, then $\gamma_{T}^{*}$ can be found by solving following equation numerically

$$
\gamma_{T}^{*}=\arg _{\gamma_{T}} \max \left(Q_{m}^{*}, Q_{f}^{*}\right), \quad Q_{m}^{*} \leq 0.1, Q_{f}^{*} \leq 0.1 .
$$

The maximum normalized saved energy/bandwidth is then given by $(1-g) \hat{p}^{*}$, where $\hat{p}^{*}=\int_{0}^{\gamma_{T}^{*}} f_{\gamma}(x) d x$.

\section{NUMERICAL RESULTS}

Figure 3 shows the fused miss-detection probability $\left(Q_{m}\right.$ and $\left.Q_{m}^{*}\right)$ and the fused false alarm probability $\left(Q_{f}\right.$ and $\left.Q_{f}^{*}\right)$ at a R-CPE as function of the number of the S-CPEs that are instructed to sense the spectrum, i.e., $N$. Rayleigh fading channel with an average SNR $(\bar{\gamma})$ of $10 \mathrm{~dB}$ is assumed, and the channel is assumed being occupied by the primary users over $80 \%$ of the time, i.e., $P_{1}=0.8$ and $P_{0}=0.2$. The SNR threshold $\left(\gamma_{T}\right)$ is set to $5 \mathrm{~dB}$ and $u$ is set to $10 . g$ is set to 0.2 , that is to say there are $20 \%$ of the S-CPEs participating sensing have been allocated upstream bandwidth. At each S-CPE, the energy threshold $\lambda$ is so set that the fused error probability is minimized. Clearly, it can see that when the number of the S-CPEs increases, the spectrum sensing performance becomes better. When energy/bandwidth-saving SCC scheme is applied for a given $N$, false alarm probability becomes lower while miss-detection probability becomes higher, however, the fused error probability changes very little. When $N$ equals to 10 , $Q_{m}, Q_{m}^{*}, Q_{f}$ and $Q_{f}^{*}$ are all lower than $10 \%$, meaning that the CSS approach meets the requirement of 802.22 system in both cases with and without applying the energy/bandwidth-saving scheme. Since $\gamma_{T}$ is set to $5 \mathrm{~dB}$, when the energy/bandwidthsaving method is applied, about $22 \%$ energy/bandwidth can be saved.

Figure 4 shows the fused miss-detection probability $\left(Q_{m}\right.$ and $\left.Q_{m}^{*}\right)$ and the fused false alarm probability $\left(Q_{f}\right.$ and $\left.Q_{f}^{*}\right)$ at a R-CPE as function of the threshold of SNR $\left(\gamma_{T}\right)$. Other parameters setting are same as above while $N$ is set to 10 and 20 for comparison. $Q_{m}, Q_{f}$ and $Q_{e}$ keep unchange with $\gamma_{T}$ since all Group-2 S-CPEs detecting $H_{1}$ need to report. As observed, the fused false alarm probability after enery/bandwidth-saving CSS is applied $\left(Q_{f}^{*}\right)$ increases with the SNR threshold $\left(\gamma_{T}\right)$ since when less Group-2 S-CPEs detecting $H_{1}$ report to a R$\mathrm{CPE}$, the success reporting probability is higher due to less contention, while the fused miss-detection probability $\left(Q_{m}^{*}\right)$ decreases with the SNR threshold due to higher success report 


$$
\begin{aligned}
& Q_{m_{2}}^{*}=\sum_{I=0}^{(1-g) N}\left(\begin{array}{c}
(1-g) N \\
I
\end{array}\right)\left(p_{d} \hat{p}\right)^{I}\left(1-p_{d}\right)^{[(1-g) N-I]} \\
& +\sum_{M=2}^{(1-g) N}\left(\begin{array}{c}
(1-g) N \\
M
\end{array}\right)\left\{p_{d}(1-\hat{p})\left[1-p_{t}(M)\right]\right\}^{M}\left\{\begin{array}{c}
(1-g) N-M \\
\sum_{I=0}\left(\begin{array}{c}
(1-g) N-M \\
I
\end{array}\right)\left(p_{d} \hat{p}\right)^{I}\left(1-p_{d}\right)^{[(1-g) N-M-I]}
\end{array}\right\} \\
& Q_{f_{2}}^{*}=1-\sum_{J=0}^{(1-g) N}\left(\begin{array}{c}
(1-g) N \\
J
\end{array}\right)\left(p_{f} \hat{p}\right)^{J}\left(1-p_{f}\right)^{[(1-g) N-J]} \\
& -\sum_{L=2}^{(1-g) N}\left(\begin{array}{c}
(1-g) N \\
L
\end{array}\right)\left\{p_{f}(1-\hat{p})\left[1-p_{t}(L)\right]\right\}^{L}\left\{\begin{array}{c}
(1-g) N-L \\
\sum_{J=0}^{(9)}\left(\begin{array}{c}
(1-g) N-L \\
J
\end{array}\right)\left(p_{f} \hat{p}\right)^{J}\left(1-p_{f}\right)^{[(1-g) N-L-J]}
\end{array}\right\}
\end{aligned}
$$

probability when less Group-2 S-CPEs report. For $N=10$, when the SNR threshold $\gamma_{T}$ is higher than $8.5 \mathrm{~dB}, Q_{f}^{*}$ becomes higher than $10 \%$, meaning that the allowed maximum SNR threshold $\left(\gamma_{T}^{*}\right)$ for $N=10$ is about $8.5 \mathrm{~dB}$. For $N=20, \gamma_{T}^{*}$ is more than $10 \mathrm{~dB}$.

Figure 5 shows the normalized saved energy/bandwidth as function of the SNR threshold when the energy/bandwidthsaving scheme is applied. $g$ is set to 0.2 and $P_{1}=0.8$ and $P_{0}=0.2$. Rayleigh fading channel with average SNR of $10 \mathrm{~dB}$ is assumed. It can be seen that the saved energy/bandwidth increases rapidly with the SNR threshold. When $\gamma_{T}$ equals to 8.5 $\mathrm{dB}$, more than $40 \%$ of energy/bandwidth can be saved, while for $\gamma_{T}$ more than $10 \mathrm{~dB}$, more than $50 \%$ of energy/bandwidth can be saved.

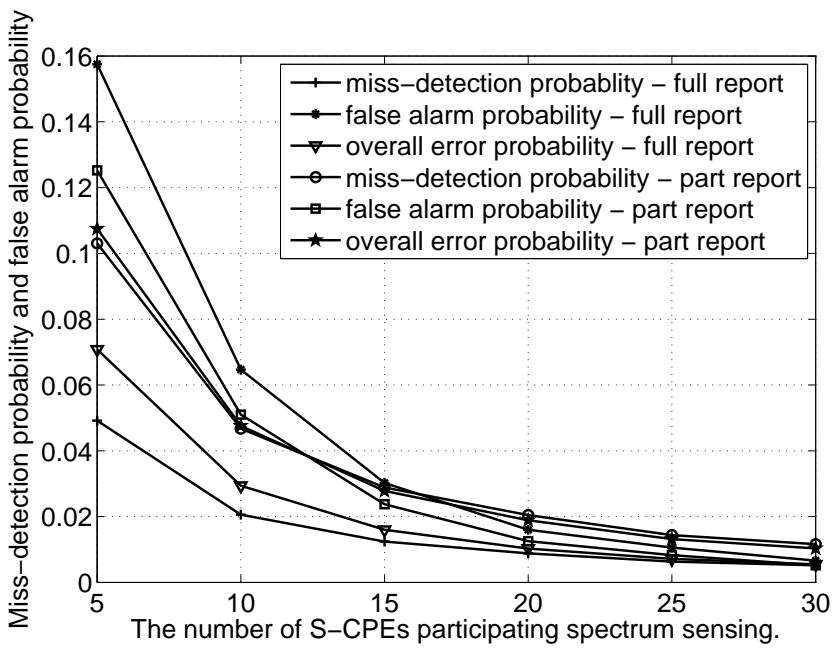

Fig. 3. Fused miss-detection probability and fused false alarm probability at a R-CPE as function of the number of S-CPEs participating spectrum sensing Rayleigh fading channel with $\bar{\gamma}$ of $10 \mathrm{~dB} .\left(P_{1}=0.8, P_{0}=0.2, \gamma_{t}=5 \mathrm{~dB}\right.$. $)$

In above, due to the performance limitation of energy detection, the assumed average SNR is relatively high. For other high performance sensing technology, relatively lower average SNR can be assumed and investigated. For example, feature detection spectrum sensing can detect PU signal with SNR lower than $-20 \mathrm{~dB}$, and at higher detected PU signal SNR, better performance can be achieved [16], [17], therefore

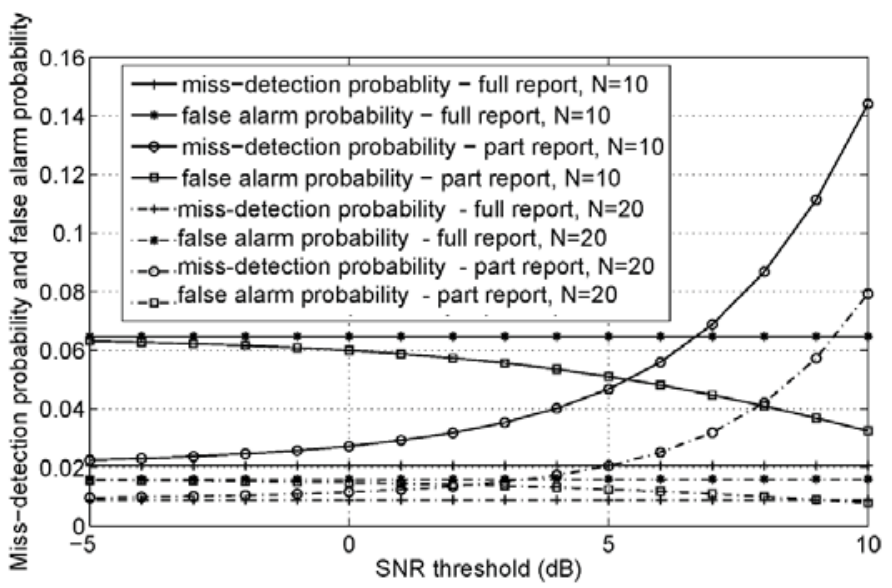

Fig. 4. Fused miss-detection probability and fused false alarm probability at a R-CPE as function of the instantaneous SNR threshold. Rayleigh fading channel with $\bar{\gamma}$ of $10 \mathrm{~dB}$. $\left(P_{1}=0.8, P_{0}=0.2\right)$

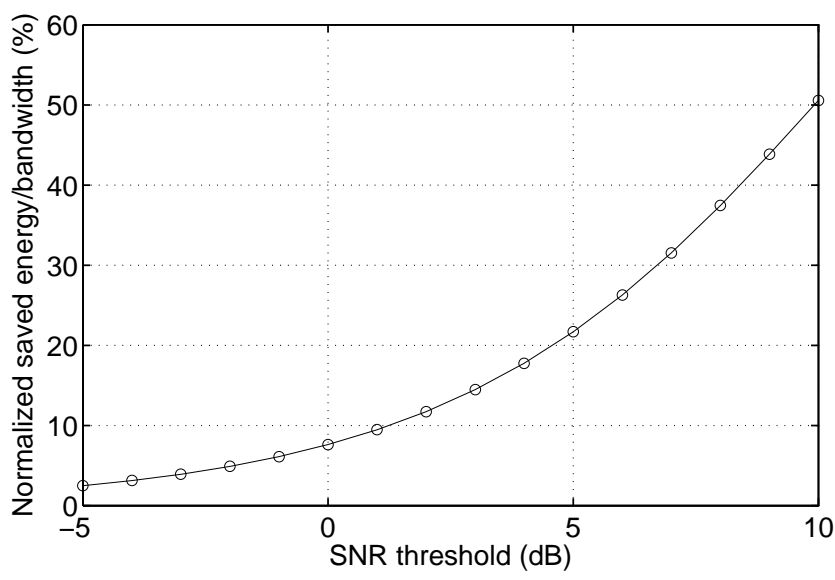

Fig. 5. Normalized saved energy/bandwidth as function of the instantaneous SNR threshold. Rayleigh fading channel with $\bar{\gamma}$ of $10 \mathrm{~dB}$. $\left(P_{1}=0.8, P_{0}=\right.$ $0.2)$

same mechanism proposed in this study can be applied, i.e., in each round spectrum sensing, the S-CPEs detecting SNR lower than a threshold SNR can be excluded from reporting their sensing results to a R-CPE and then the system overall energybandwidth can be saved. 


\section{CONCLUSION}

IEEE p802.22b engages two-hop network structure which each R-CPE connecting with an MR-BS and a great number of S-CPEs requiring low-cost and power/bandwidth-saving designs. An energy/bandwidth saving CSS scheme is proposed for $\mathrm{p} 802.22 \mathrm{~b}$ to meet the design requirement of the S-CPEs while still maintain the sensing performance. With the proposed scheme, when an S-CPE without upstream bandwidth detects the presence of PU, it reports to a R-CPE for fusion only if the detected SNR is above than a pre-defined SNR threshold. Numerical results show that with the proposed CSS, the fused miss-detection probability becomes higher and the fused false alarm probability becomes lower. It is possible to save more than $40 \%$ to $50 \%$ energy/bandwidth when 10 or 20 $\mathrm{S}$-CPEs are instructed to sense a fading channel. Although energy detection is considered in this study for simplicity in analysis, the proposed principle may be applied to other sensing technologies with higher performance.

\section{REFERENCES}

[1] IEEE Standard for Information Technology-Telecommunications and information exchange between systems Wireless Regional Area Networks (WRAN)łSpecific requirements, Part 22: Cognitive Wireless RAN Medium Access Control (MAC) and Physical Layer (PHY) Specifications: Policies and Procedures for Operation in the TV Bands, IEEE Std 802.22-2011, IEEE-SA Standards Board, 2011

[2] PAR of the IEEE Pp802.22b - Amendment to the IEEE Std-802.222011(TM): Enhancements for Broadband Services and Monitoring Applications, http://www.ieee802.org/22/Pp802.22b_PAR_Approved.pdf

[3] H.N. Pham, Y. Zhang, P.E. Engelstad, T. Skeie, and F. Eliassen, "Energy minimization approach for optimal cooperative spectrum sensing in sensor-aided cognitive radio networks", WICON 2010, pp.1-9, Singapore, Mar 2010

[4] E. Peh and Y.C. Liang, "Optimization for cooperative sensing in cognitive radio networks", IEEE WCNC 2007, pp. 27-32, Hong Kong, Mar 2007

[5] F.D. Fadel, Mohamed-Slim Alouini, and K.S. Marvin, "On the energy detection of unknown signals over fading channels", IEEE ICC'03, pp. 3575 - 3579, vol.5, Anchorage, AK, USA, May 2003

[6] T. Yücek and H. Arslan, "A survey of spectrum sensing algorithms for cognitive radio applications", IEEE Commun. Surveys \& Tutorials, vol. 11, no. 1, first quater 2009, pp.116-130

[7] K.B. Letaief and W. Zhang, "Cooperative communications for cognitive radio networks", Proceeding of the IEEE, Vol. 97, No. 5, May 2009

[8] Y. Zhang, J. Xiang, X. Qin, and G. E. Øien, "Optimal sensing cooperation for spectrum sharing in cognitive radio networks", European Wireless 2009, pp. 216-221, Denmark, May 2009

[9] W.-B. Chien, C.-K. Yang, and Y.-H. Huang, "Energy-saving cooperative spectrum sesning processor for cognitive radio system", IEEE Trans. Circuits Syst. I, Reg. papers, vol. 58, no. 4, Apr 2011, pp.711-723

[10] R. Deng, J. Chen, C. Yuen, P. Cheng, and Y. Sun, "Energy-efficient cooperative spectrum sensing by optimal scheduling in sensor-aided cognitive radio networks", IEEE Trans. Veh. Technol., vol. 61, No. 2, Feb 2012, pp. 716-725

[11] M. Najimi, A. Ebrahimzadeh, S.M. H. Andargoli, and A. Fallahi, "A novel method for energy-efficient cooperative spectrum sensing in cognitive sensor networks", IST'2012, pp. 255-260, Tehran, Nov 2012

[12] W. Zhang, R.K. Mallik, and K.B. Letaief, "Optimization of cooperative spectrum sensing with energy detection in cognitive radio networks", IEEE Tran. Wireless Commun., Vol. 8, No. 12, pp. 5761-5766, Dec 2009

[13] H. Tang, "Some physical layer issues of wide-band cognitive radio systems", IEEE Dyspan 2005, pp. 151-159, USA, Nov 2005

[14] A. Wiesel, J. Goldberg, and Hagit Messer-Yaron, "SNR Estimation in Time-Varying Fading Channels", IEEE Tran. Commun., Vol. 54, No. 5, pp. 841-848, May 2006

[15] David R. Pauluzzi and Norman C. Beaulieu, "A Comparison of SNR Estimation Techniques for the AWGN Channel", IEEE Tran. Commun., Vol. 48, No. 10, pp. 1681-1691, Oct 2000
[16] Chunyi Song and Hiroshi Harada, "Proposal and Hardware Implementation of a Partial Bandwidth Based Feature Detection Method for Sensing under Adjacent Channel Interference," IEEE Transactions on Wireless Communications, Vol.12, Issue 11, pp.5444-5453, Nov. 2013

[17] Chunyi Song, Matsumura Takeshi and Hiroshi Harada,"A Prototype of TV White Space Spectrum Sensing and Power Measurement," IEICE Trans. on Communications, VOL.E97-B, NO.2, pp 314-325, Feb. 2014 